\title{
考虑真实能量来源的大洋垂直湍流混合系数气候 数据集
}

\author{
张宇 ${ }^{(1)}$, 林一骅 ${ }^{(* *}$, RuiXin HUANG ${ }^{\circledR}$ \\ (1) 国家海洋环境预报中心, 北京 100081 ; \\ (2) 中国科学院大气物理研究所, 大气科学和地球流体力学数值模拟国家重点实验室, 北京 100029 ; \\ (3) Department of Physical Oceanography, Woods Hole Oceanographic Institute, Woods Hole, MA 02543, USA \\ * 联系人, E-mail: linyh@lasg.iap.ac.cn
}

收稿日期: 2013-09-23; 接受日期: 2013-12-04; 网络版发表日期: 2014-08-05

国家自然科学基金项目(批准号: 41175058)资助

\begin{abstract}
摘要 利用 ERA Interim 再分析风和海浪资料、SODA 三维温盐流资料、WOA09 海 洋温盐客观分析资料计算了风和潮汐引起的大洋垂直湍流混合系数, 并从外部风能量 输入、混合引起的重力位能改变、人为限定混合系数上限、不考虑外部能量输入的 $\mathrm{K}$ 理论参数化方案角度分别讨论了大洋垂直混合的能量守恒问题. 研究表明: 平均化资料 和混合系数限定都会造成显著的能量不守恒, 二者可造成高达 $90 \%$ 的能量损失及能量 输入周期律的显著改变. 风在南大洋有巨大的能量输入至海洋上层湍流中, 潮汐在深海 山脉附近也有巨大的能量输入. 本文认为严格考虑外部风和潮汐能量输入对于海洋长 期气候模拟是必要的, 在目前模式技术水平下恰当的使用本文计算出的混合系数集是 一个快速经济的选择.
\end{abstract}

\section{关键词 \\ 能量守恒 \\ 谱分析 \\ 湍流混合参数化方案 \\ 垂直湍流混合系数}

大洋中的垂向跨等密度面湍流混合(简称混合, 下同)一直是物理海洋研究的热点, 也是海洋数值模 拟长期以来的研究难点. 由于数值模式在垂向上的 网格距尚不能满足湍流直接模拟的需要, 因此湍流 混合参数化成为了解决这一问题的必要方法. $\mathrm{K}$ 理论 模型是海洋模式中应用最广泛的一类参数化方案, 其核心思想是将各类湍流的效应量化为垂直湍流混 合系数 $\mathrm{K}$ (混合系数, 下同), 并将 $\mathrm{K}$ 倍乘平均量的梯 度来模拟湍流对平均场的影响. $\mathrm{K}$ 理论参数化方案的 代表有 PP(Pacanowski 和 Philander, 1981)、MY(Mellor
和 Yamada, 1982)、KPP(K-Profile Parameterization (Large 等, 1994)). 这些方案无论是从梯度理查森数 来考虑湍流发生的难易程度(PP), 还是采用一系列复 杂的高阶预报方程(MY), 或者考虑非局地项以及温 盐双扩散效应(KPP), 其最重要的目的都是为了准确 的估计混合系数.

混合系数的改变可以极大地影响数值模式的模 拟结果，中外学者用不同的模式和实验设计都证明 了这一点(Boos 等, 2004; Bryan, 1987; Huang 等, 2007; Samelson, 1998; 雷雯和杨海军, 2008; 刘玲玲和黄传

中文引用格式: 张宇, 林一骅, Huang R X. 2014. 考虑真实能量来源的大洋垂直湍流混合系数气候数据集. 中国科学: 地球科学, 44: 2089-2100

英文引用格式: Zhang Y, Lin Y H, Huang R X. 2014. A climatic dataset of ocean vertical turbulent mixing coefficient based on real energy sources. Science China: Earth Sciences, doi: 10.1007/s11430-014-4904-6 
江, 2008), 因此混合系数的改变直接关系到模拟结果 的物理意义及科学上的合理性, 其给定是需要慎重 考虑的. 而 $\mathrm{K}$ 理论参数化方案中普遍具有大量的可 调节参数, 有的参数物理意义很模糊, 如 PP 中的梯 度理查森数倍率 $\alpha$; 有的则具有较大的人为随意性, 如 KPP 中的混合系数垂直分布形态函数. 这些参数 的调节都可以引起混合系数的显著改变, 而如何准 确的给定这些参数一直存在争议.

从能量的角度看, $\mathrm{K}$ 理论参数化方案都没有很好 的考虑能量的守恒性. 根据 Huang(1999)和黄瑞新 (1998)的研究, 混合是需要外部能量维持的, 湍流混 合参数化方案应当从外部能量输入和局地湍流发生 难易程度两方面来共同确定混合系数, 而目前的 $\mathrm{K}$ 理论参数化方案大都没有很好的考虑外部能量输入. 大洋中的外部能量主要有风和潮汐, 目前二者都可 以通过参数化的方式计算其输入至海洋中的能量 (Craig 和 Banner, 1994; Laurent等, 2002), 并进一步计 算出混合系数(Osborn, 1980). 但是, 风浪致混合系 数的参数化需要高时空分辨率的海浪模式来计算所 需的风应力和海浪高度; 潮汐致混合系数的参数化 也需要高精度的潮汐模式和地形数据来计算内潮破 碎输入海洋的湍流动能. 目前的海洋数值模式尤其 是用于长时间气候态积分的大洋环流模式很难达到 这样高的分辨率要求. 利用高精度的海浪、潮汐模式 和观测数据, 计算出风和潮汐产生混合系数的气候 态数据集, 并直接将气候态的混合系数数据应用至 大洋环流模式中是解决这一难题的一个简单有效的 办法. 本文将利用高时间分辨率风应力、海浪再分析 数据、海洋三维温盐流客观分析数据和全球潮汐湍流 动能耗散数据, 结合前人发表的观测结果, 初步整合 出一套可用于大洋环流模式的全球混合系数气候 态数据集并同 $\mathrm{K}$ 理论参数化方案对比讨论其能量守 恒性.

\section{1 数据与计算方法}

\section{1 数据来源}

本文使用的数据主要有四种.

(1) 由美国国家海洋数据中心 (National Oceanographic Data Center, NODC)提供的 WOA09 (World
Ocean Atlas 2009)气候态海洋温盐客观分析数据. 该 资料的覆盖范围为 $0.5^{\circ} \mathrm{E} \sim 359.5^{\circ} \mathrm{E}, 89.5^{\circ} \mathrm{S} \sim 89.5^{\circ} \mathrm{N}$, 水 平分辨率为 $1^{\circ} \times 1^{\circ}$, 垂向 $0 \sim 5500 \mathrm{~m}$ 分为 33 层.

(2) 由欧洲中期天气预报中心(The European Centre for Medium-Range Weather Forecasts, ECMWF) 提供的 ERA Interim(ECMWF Re-analyses Interim data) 资料中的海表风应力和海表面波浪高度数据. 该资 料的覆盖范围为 $0^{\circ} \sim 358.5^{\circ} \mathrm{E}, 90^{\circ} \mathrm{S} \sim 90^{\circ} \mathrm{N}$, 空间分辨 率为 $1.5^{\circ} \times 1.5^{\circ}$, 时间分辨率为 $6 \mathrm{~h}$, 数据起止时间为 1979 年 1 月 1 日至 2011 年 12 月 31 日.

(3) 由美国马里兰大学提供的 SODA(Simple Ocean Data Assimilation)资料 2.1.6 版. 该资料的覆盖 范围为 $0.25^{\circ} \mathrm{E} 359.75^{\circ} \mathrm{E}, 75.25^{\circ} \mathrm{S} \sim 89.25^{\circ} \mathrm{N}$, 水平分辨 率为 $0.5^{\circ} \times 0.5^{\circ}$, 垂向 5 5375 $\mathrm{m}$ 分为 40 层, 时间分辨 率为月平均, 数据起止时间为 1979 年 1 月至 2008 年 12 月.

(4) 美国 Woods Hole海洋研究所 Steven R. Jayne 于 2008 年计算的全球大洋潮汐湍流能量耗散气候态 平均数据. 该资料的覆盖范围为 $0.5^{\circ} \mathrm{E} 360^{\circ} \mathrm{E}$, $83^{\circ} \mathrm{S} \sim 83^{\circ} \mathrm{N}$, 水平分辨率为 $0.5^{\circ} \times 0.5^{\circ}$.

为满足本文研究需要, 对原始数据做了预处理 工作. 本文用 The Gibbs Sea Water(GSW)Oceanographic Toolbox of TEOS-10 标准程序将 WOA09 和 SODA 中的现场温度转换成位温 (以海平面为参考面), 对 WOA09 资料预先做重力调整，修正一些上下不稳 定的区域(需调整的格点数约占全部格点数的 $0.02 \%$ ), 并将其中的实用盐度转换为绝对盐度, 进一步计算 了每一层的现场密度和以海平面为参考面的位密 $\sigma_{0}$, 用于计算海洋上层的浮力频率.

\section{2 计算方法}

本文的计算过程主要有三种.

(1) 风和海浪引起的海洋上层湍流动能耗散率 及风输入海洋中的能量. 计算方案主要参照 Baumert(2005)的方案，将海洋上层分为波浪混合层、 湍流混合层和壁面律层分别计算.波浪混合层计算 公式为

$$
\varepsilon(z)=\frac{150 u_{*}^{3} z_{0}^{3 / 2}}{\kappa\left(z+z_{0}\right)^{5 / 2}}, \quad z<z_{d}, \quad z_{d}=25 H_{s} .
$$

湍流混合层计算公式为 
$\varepsilon(z)=\frac{150 u_{w_{w}}^{3} z_{0}^{3 / 2}}{\kappa\left(z+z_{0}\right)^{5 / 2}}+\left(\frac{u_{w_{w}}^{3}}{\kappa\left(z+z_{0}\right)}\right)^{m}, \quad z<z_{b}, \quad z_{b}=100 H_{s}$.

式中, $u_{*_{w}}=\sqrt{\tau / \rho_{w}}, \tau$ 是海表面风应力, $\rho_{w}$ 是海水密 度, $z_{0}$ 按照 Charnock(1955)和 Soloviev 等(2003)的估计 约为 $a u_{*_{w}}^{2} / g, a \approx 150000, H_{s}$ 为海表面特征波高, $\kappa$ 为卡曼常数, 本文取 $0.4, \mathrm{~m}$ 为可调节的经验系数, 本 文取 $1.02, z_{d}$ 的取值参照 Terray 等(1996)的研究, $z_{b}$ 的 取值参照 Kocsis 等(1999)的研究. 湍流混合层以下可 以视为风浪无法影响到的区域, 不进行参数化处理. 由此只要确定 $\tau$ 和 $H_{s}$, 即可由式(1)和(2)计算出局地 风浪致湍流动能耗散率 $\varepsilon$ 的垂直分布. 获得 $\varepsilon$ 后, 进 一步乘以海水质量即可得风输入海洋中的能量.

(2) 由湍流动能耗散率参数化混合系数. 计算方 案采用 Osborn(1980)提出的参数化方案:

$$
K=\frac{R_{f} \varepsilon}{\left(1-R_{f}\right) N^{2}},
$$

式中, $K$ 为混合系数, $R_{f}$ 为通量理查森数, $N^{2}$ 为浮力频 率的平方. 当 $R_{f}$ 取临界值 0.15 时, 则有

$$
K \leqslant \frac{0.15 \varepsilon}{0.85 N^{2}}<0.2 \frac{\varepsilon}{N^{2}},
$$

式中, 0.2 可以认为相当于混合效率 $\alpha$, 表示可转化为 湍流混合的最大能量比率. Osborn(1980)认为就大洋 平均意义而言, 取 0.2 是合理的. Ivey 等(1991)和 Moum(1996)及其他大量的研究认为混合效率不应是 一个常数, 取值应在 0.1 0.4. Lozovatsky 等(2006)认 为 $\alpha$ 和理查森数有关, Lozovatsky 等(2013)的研究表明, 混合效率 $\alpha$ 与理查森数间存在近似 $\alpha=0.005+$ $1.7 R i-1.1 R i^{2}$ 的关系, 其中 $R i<1$ 为理查森数. 由此只 要获知 $\alpha, \varepsilon$ 和 $N^{2}$ 就可计算出局地混合系数. 但是 $R i$ 的计算需要用高频的实际观测资料, 任何平均化的 海洋资料都会消除湍流运动, 导致不合实际状况的 $R i$ 计算结果. 根据 Oakey 等(2004)的综合研究, 平均 气候态意义下 $\alpha$ 取 0.2 仍是合理的, 因此本文计算仍 采用全球均一值 0.2 替代混合效率. 实际计算中为避 免过小的 $N^{2}$ 带来巨大的混合系数, 可将 $N^{2}$ 用 $N^{2}+\Omega^{2}$ 取代, 其中 $\Omega$ 为地球自转和公转的角频率之和, 为 $7.2921 \times 10^{-5} \mathrm{~s}^{-1}$, 这样做仅仅是出于保证数值模式中 $K$ 理论参数化方案计算的稳定性, 实际大洋中 $N^{2}<\Omega^{2}$ 的格点较少, 约占总格点数的 $3 \%$. 在这些区 域 $K$ 理论参数化不再具备物理意义, 需要数值模式
的其他模块对这些区域进一步处理.

(3) PP 参数化方案计算热带地区海洋上层混合 系数. 参数化方案详见 Pacanowski 等(1981), 其核心 公式为

$$
\begin{aligned}
& K=\frac{K_{0}}{\left(1+5 R_{i}\right)^{3}}+K_{b}, \\
& R_{i}=\frac{\beta g \theta_{z}}{U_{z}^{2}+V_{z}^{2}}, \quad \beta \sim 8.75 \times 10^{-6}(\theta+9),
\end{aligned}
$$

式中, $K_{0}$ 是最大混合系数, 本文取 $5 \times 10^{-3} \mathrm{~m}^{2} \mathrm{~s}^{-1}, K_{b}$ 是背景混合系数, 本文取 $1 \times 10^{-5} \mathrm{~m}^{2} \mathrm{~s}^{-1} . R_{i}$ 是梯度理 查森数, 当 $R_{i}<0$ 时, $K$ 直接取最大值 $K_{0} . \beta$ 是热扩散系 数, $\theta$ 是位温, $U$ 是海水纬向速度, $V$ 是海水经向速度, $\mathrm{g}$ 是重力加速度, 本文取 $9.8 \mathrm{~m}^{2} \mathrm{~s}^{-1}$.

\section{2 平均态数据与瞬态数据计算差异分析}

目前大部分大洋环流模式都用月平均的风应力 等外部强迫来驱动, 这对于模拟海浪而言是远远不 够的. 因此通过外部海浪模式计算出风浪对海洋上 层混合的影响, 再将其引入大洋环流模式是在模式 中再现风浪混合效应的可行办法. 对于建立气候态 的混合系数数据, 平均化是必要的手段. 鉴于参数化 方案存在较强的非线性关系, 平均风应力和浪高参 数化的结果可能和瞬时风应力和浪高参数化的结果 有较大不同. 为此, 本文分别计算了 $6 \mathrm{~h}$ 一次(未平均 的瞬时资料)、日平均和月平均的风应力和浪高输入 大洋中的能量(图 1). 由于混合系数和混合增加的重 力位能基本成线性正比关系(张宇等, 2013), 因此本 文也计算了混合增加的重力位能(图 2)以说明不同风 场资料对混合系数参数化的影响. 风浪数据采用 ERA Interim, 浮力频率用 WOA09 数据计算, 数据说 明详见上文. 风输入海洋中的能量计算方法见小节 1.2, 混合增加重力位能的算法参照张宇等(2013).

由于数据量巨大, 此处仅选择 2010 年的计算结 果说明, 实际上其他时段也有类似的结论. 由图 1 可 知, $6 \mathrm{~h}$ 瞬时、日平均和月平均三种风应力和浪高计算 出的结果差异巨大, 随着平均化周期的加长, 风输入 海洋的能量和风致湍流增加的重力位能都不断衰减. $6 \mathrm{~h}$ 瞬时资料计算出的能量输入最高超过 $80 \mathrm{TW}$, 最 低也有 $30 \mathrm{TW}$, 平均值约为 $49.6 \mathrm{TW}$; 日平均资料计 算出的能量输入最高为 $70 \mathrm{TW}$, 最低为 $25 \mathrm{TW}$, 平均 值约为 $43.8 \mathrm{TW}$; 月平均资料计算出的能量输入最高 
只有 $30 \mathrm{TW}$, 最低约为 $22 \mathrm{TW}$, 平均值只有约 26.6 TW. 相对于瞬时数据, 日平均风场输入的能量平均 衰减了 $11.74 \%$, 月平均风场输入的能量衰减可达 $46.2 \%$.

混合增加的重力位能和风输入能量有完全一致 的特征(图 2), 随着资料平均化程度的增加能量不断
衰减. 此外, 重力位能变化的瞬时、日平均、月平均 归一化标准差分别为 $0.1712,0.1852$ 和 0.29 , 只比风 输入能量对应的 $0.1684,0.1825$ 和 0.2855 略大一点, 在统计意义上并不显著, 这在另一角度说明了密度 的非线性作用在全球这能量积分中体现不明显.

从能量守恒的角度看, 用平均化的风应力驱动

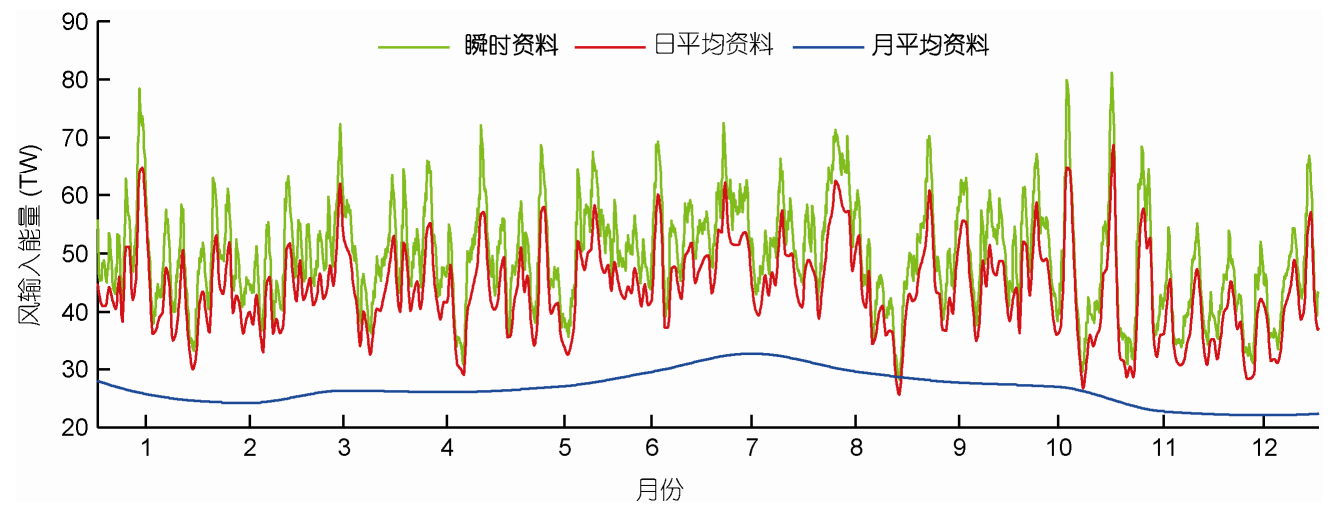

图 1 每 $6 \mathrm{~h}$ 瞬时资料、日平均资料和月平均资料计算的风输入大洋中能量

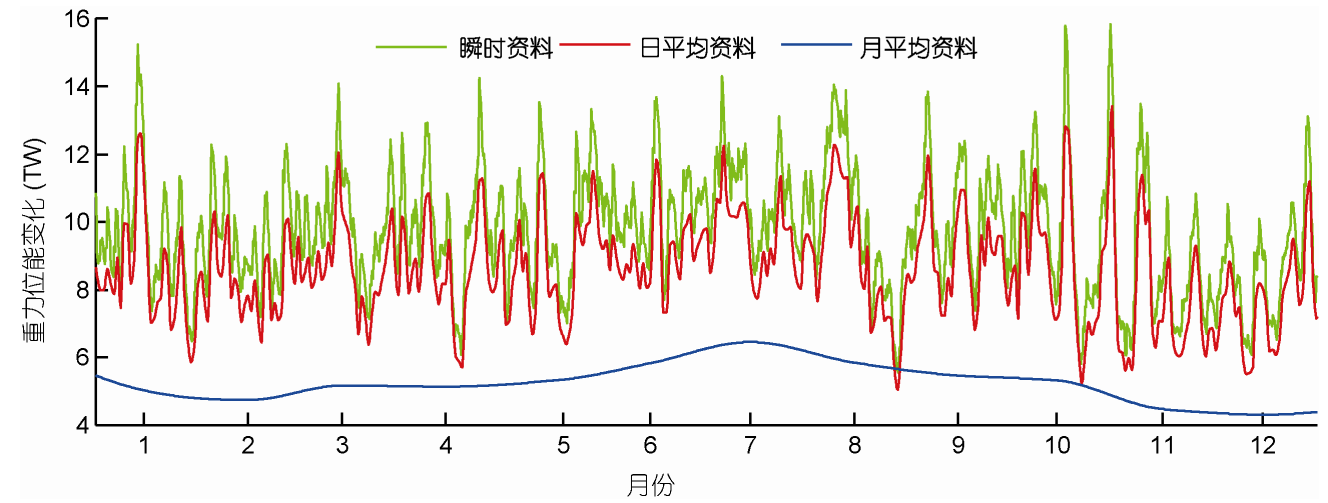

图 2 图 1 中三种风输入能量引起的湍流混合致重力改变

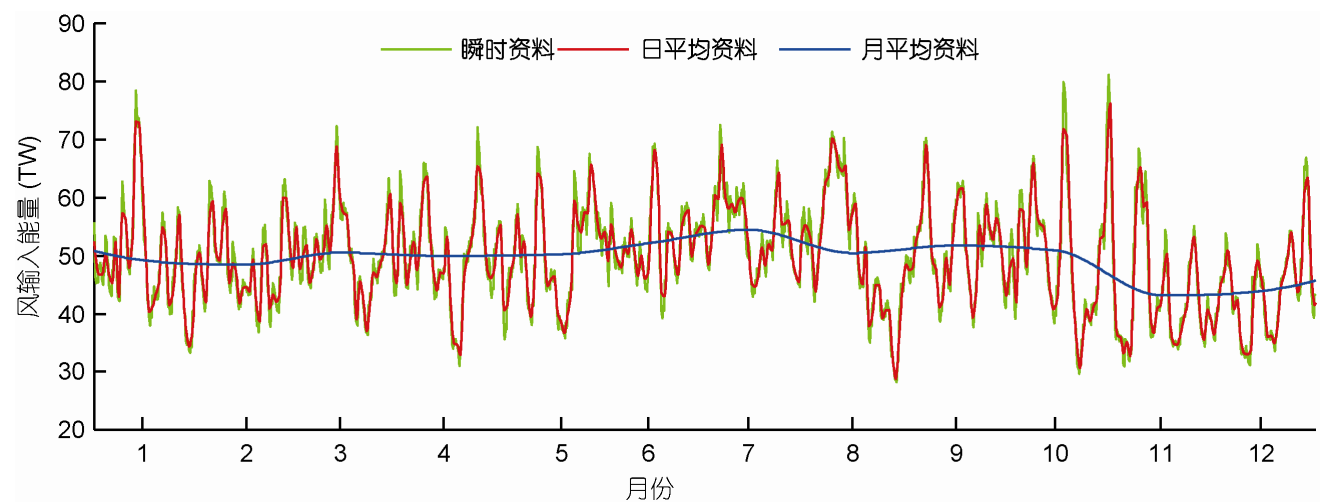

图 3 风输入大洋中的能量

先用每 $6 \mathrm{~h}$ 瞬时资料计算瞬时能量输入再进行日平均和月平均 
大洋环流模式会造成极大的能量损失, 但如果先用 瞬时资料计算出瞬时的能量输入和混合系数, 然后 再做气候平均则会极大的避免能量不守恒, 本文称 为后平均法 (图 3 和 4).

由图 3 和 4 可知, 对能量输入和混合系数的后平 均结果很好的保持了能量守恒性. 瞬时、日平均、月 平均风输入的能量平均值几乎完全一致, 分别为 49.6389, 49.6389 和 $49.6260 \mathrm{TW}$; 混合增加重力位能 的平均值分别为 $9.7466,9.7463$ 和 $9.7427 \mathrm{TW}$, 三者 也相当一致. 因此从能量守恒的角度看, 用瞬时资料 计算出湍流动能耗散和混合系数后, 再平均化得气 候态数据并应用于长期气候模拟研究是合理的. 不 过从图 3 和 4 中也可以看出, 季节尺度以下的能量周 期变化在月平均中几乎被完全消除, 因此用月平均 气候混合系数模拟短时间内的大洋环流可能会抹杀 中小尺度运动的贡献.

\section{3 湍流混合系数的限定}

在实际混合系数参数化中, 由于大洋中存在负 的或非常小的 $N^{2}$ 和 $R_{i}$, 根据式(4), 此时混合系数计 算值可能非常大. 从数值模式的角度看, 过大的混合 系数容易产生积分不稳定, 因此许多应用式(4)参数 化混合系数的模式会人为设定上限, 一般取 $5 \times 10^{-3}$ $\mathrm{m}^{2} \mathrm{~s}^{-1}$. 但从能量守恒的角度看, 人为的设定上限可 能会引起能量的不守恒, 而且在有大量风能输入弱 层结海区时, 高混合系数(超过 $5 \times 10^{-3} \mathrm{~m}^{2} \mathrm{~s}^{-1}$ ) 未必在 物理上不合理. 为研究人为限定造成的能量损失, 本 文同样以 2010 年为例, 计算了设定上限时参数化混 合系数对重力位能的贡献(图 5).

由图 5 可以明显看出, 由于人为限定混合系数后 混合强度大大削弱, 其对重力位能的贡献也显著减 弱. 瞬时场由于大量的峰值波动被人为削减, 湍流能 量损失最大, 最高可超过 $90 \%$, 重力位能变化的周期

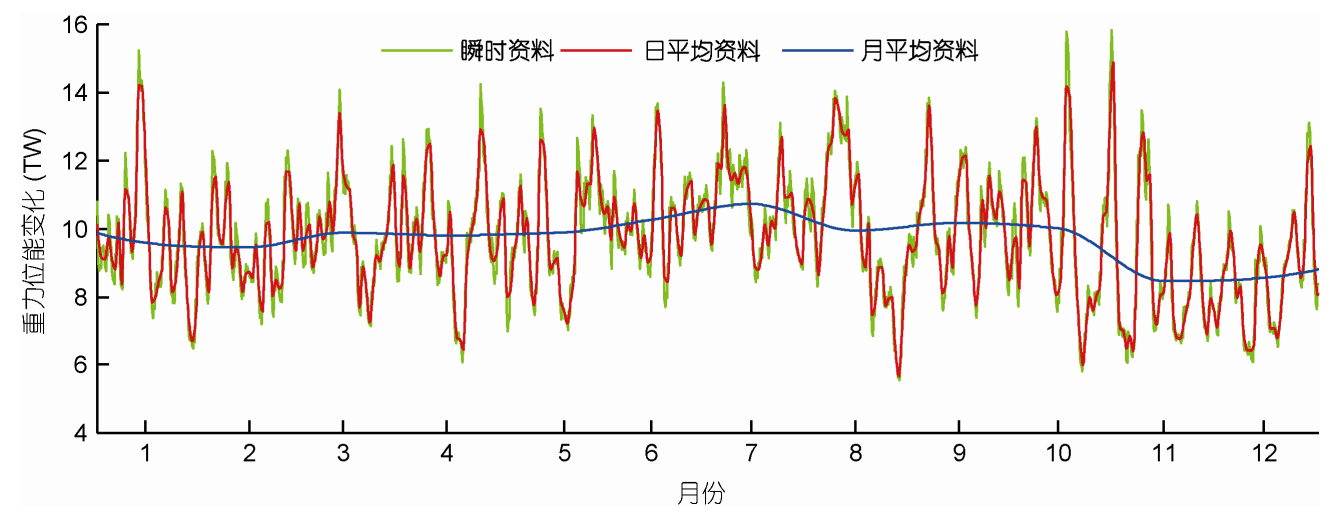

图 4 图 3 中三种风输入能量引起的湍流混合致重力改变

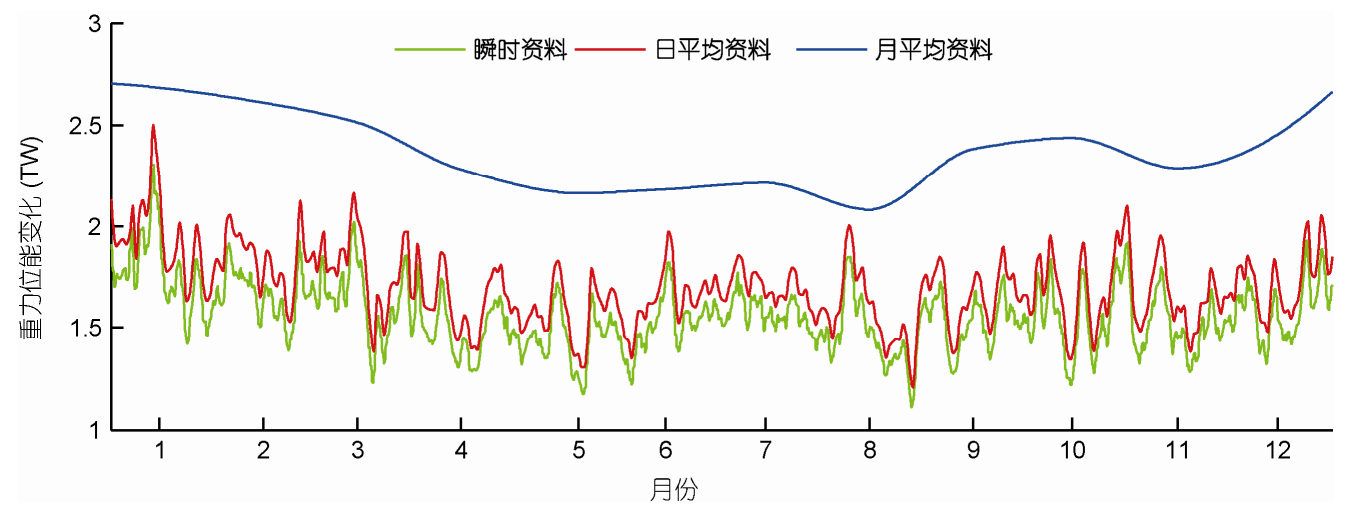

图 5 限定混合系数后湍流参数化致重力位能改变

绿线代表对图 4 中绿线所用混合系数做人为限定后的新结果, 红线对应图 4 中红线, 蓝线对应图 4 中蓝线 
运动振幅也衰减至不足 $1 \mathrm{TW}$. 平均场则因为峰值波 动相对较弱能量损失也较小, 但削弱幅度也超过了 $70 \%$.

由于地球的自转和公转，很多海洋现象具有日、 季节、年际等尺度上的周期运动特征. 为检验人为限 定混合系数对能量输入周期演变规律的影响, 本文 选取风季节变化较强的热带地区，分析了风及湍流
的能量功率谱(图 6). 图 6 中给出了能量输入的功率 谱(蓝色实线)和红噪声功率谱(红色虚线), 功率谱的 计算方法详见黄嘉佑(2004). 当能量谱线值大于噪声 谱线值时, 可以认为该周期的功率谱贡献是统计上 可信的. 从图 6 可知人为限定不仅减弱了湍流强度, 也改变了湍流能量输入的周期特征. 图 6(a)显示出风 输入的能量有统计上显著可信的周期为 6 个月的演
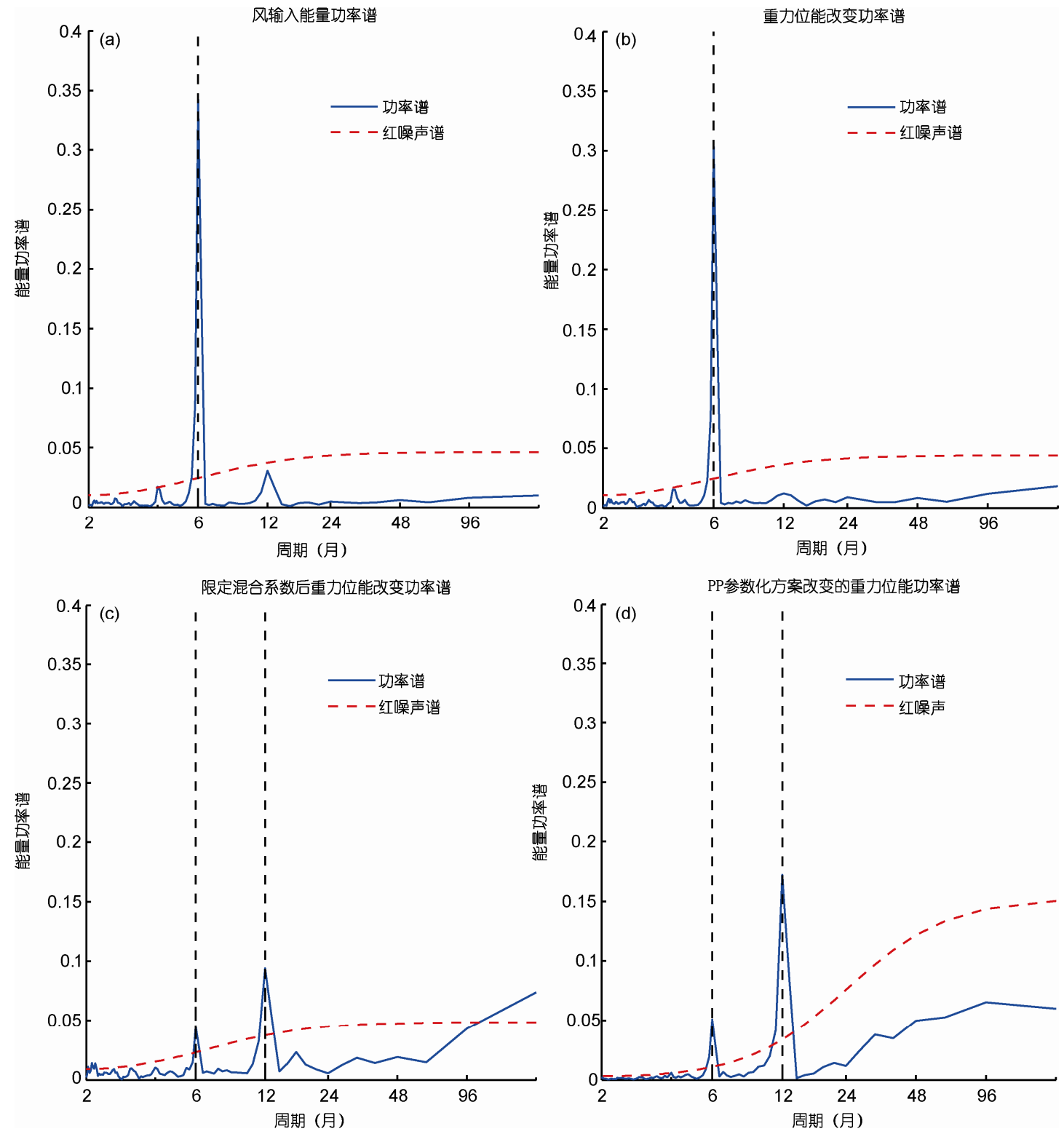

图 6 每 $6 \mathrm{~h}$ 瞬时资料计算风输入海洋湍流能量及重力位能变化的功率谱

(a) 风输入能量功率谱; (b) 未经人为限定混合系数时重力位能变化的功率谱; (c) 人为限定混合系数时重力位能变化(图 7 蓝线)的功率谱; (d) PP 参数化方案致重力位能变化(图 7 红线)的功率谱. 横坐标代表能量变化周期, 纵坐标表示每个周期上包含能量对总能量的贡献, 黑色虚线 表示该周期统计可信 
变规律, 对应的湍流增加重力位能也有显著的半年 演变周期(图 6(b)). 经过人为限定后湍流运动的周期 律被显著改变, 原本唯一的 6 个月周期混淆为 6 个月 和 12 个月两种周期律, 且前者的功率贡献被极大削 弱, 此外还有大量功率贡献转移到更低频的红噪声 特征中(图 6(c)).

\section{$4 \mathrm{~K}$ 理论参数化方案(PP) 的能量守恒性}

目前许多 $\mathrm{K}$ 理论参数化方案都没有考虑到外部 能量尤其是风和潮汐能的输入, 作为对比本文从能 量守恒的角度分析了此类参数化方案的不足. 为避 免大量可调参数带来的不确定性, 本文选取了参数 较少的 PP 方案示例分析, 该方案被证明在热带地区 有很好的模拟能力(Pacanowski 和 Philander, 1981), 方案的计算方法详见小节 1.2. 我们计算了热带地区 $\left(0.25^{\circ} \mathrm{E} 359.75^{\circ} \mathrm{E}, 30^{\circ} \mathrm{S} \sim 30^{\circ} \mathrm{N}\right) 1979$ 年 1 月至 2008 年 12 月间 PP 方案参数化的混合系数及其改变的重力位 能和相同时间空间上风混合(限定混合系数)对重力 位能的改变(图 6(d)和 7). PP 方案所需温盐流数据来 自 SODA, 风混合所需风应力及浪高数据来自 ERA Interim. 图 7 中蓝色实线为人为限定混合系数后风混 合改变的重力位能, 红色实线为 PP 方案改变的重力 位能, 黑色实线为 PP 方案相对于风混合对重力位能 影响的大小，黑色虚线为 $100 \%$ 指示线.

由图 7 可知, PP 参数化方案对重力位能的贡献甚 至小于限定混合系数后的风混合, 在平均意义下前 者只有后者的 $80 \%$. 从功率谱(图 6(d)) 可看出, PP 方 案参数化的湍流运动也具有 6 个月和 12 个月的周期 运动特征, 但是从时间演变上(图 7)则发现二者的波
动位相并不一致, 大致呈反位相. 这意味着尽管具有 相同的周期特征，但二者对热带海洋运动的影响几 乎是完全相反的. 如果和不限定混合系数时的风混 合相比, PP 方案对重力位能贡献更是衰减到几乎不 能维持大洋中的能量平衡.

\section{5 气候态混合系数数据集}

基于小节 2 和 3 的讨论, 本文建立了风致湍流混 合系数的气候态数据集, 同时利用 Simmons 等(2004) 和 Laurent 等(2002)的方法和 Steven R. Jayne 提供的 数据计算了潮汐致混合系数的气候态数据集. 基于 上文的讨论, 本文不对混合系数做人为限定, 同时保 留湍流动能耗散率以方便数据集在数值模式中的灵 活应用(图 8 11).

从图中可以看出不限定混合系数时全球大范围 内都出现非常强的湍流混合, 最高值可达 $0.5 \mathrm{~m}^{2} \mathrm{~s}^{-1}$ 以上. 风混合的高值区主要位于南大洋、北大西洋和 北太平洋; 而赤道西太平洋、赤道东太平洋、印度洋 北部、赤道东大西洋则都是低值区(图 8). 垂直方向 上赤道风混合影响深度较浅, 集中在约在 $150 \mathrm{~m}$ 以上, 在赤道中太平洋略深; 此外, 在赤道东太平洋和大西 洋温跃层之下存在一个显著的强混合区, 说明此处 风混合效应可以突破温跃层障碍(图 10). 南大洋区域 风混合最强, 可以影响到 $1000 \mathrm{~m}$ 水深处, 但超过 $5 \times 10^{-3} \mathrm{~m}^{2} \mathrm{~s}^{-1}$ 的高值区主要集中在 $150 \mathrm{~m}$ 以上, 在南 太平洋则深达 $300 \mathrm{~m}$. 同赤道类似, 南大洋在次表层 约 $300 \mathrm{~m}$ 深处也存在一个新的强混合区域(图 11). 潮 汐引起的湍流混合主要和海底地形有关, 地形复杂、 粗粘度较大的区域潮汐破碎引起的湍流也越强, 因

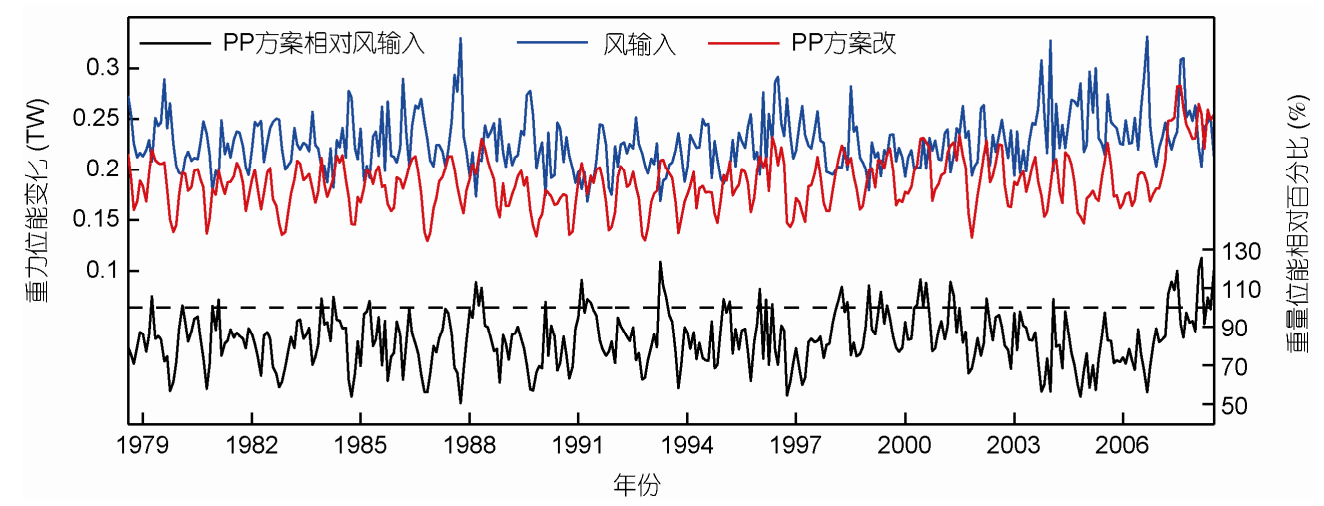

图 7 PP 参数化方案和风混合改变重力位能 


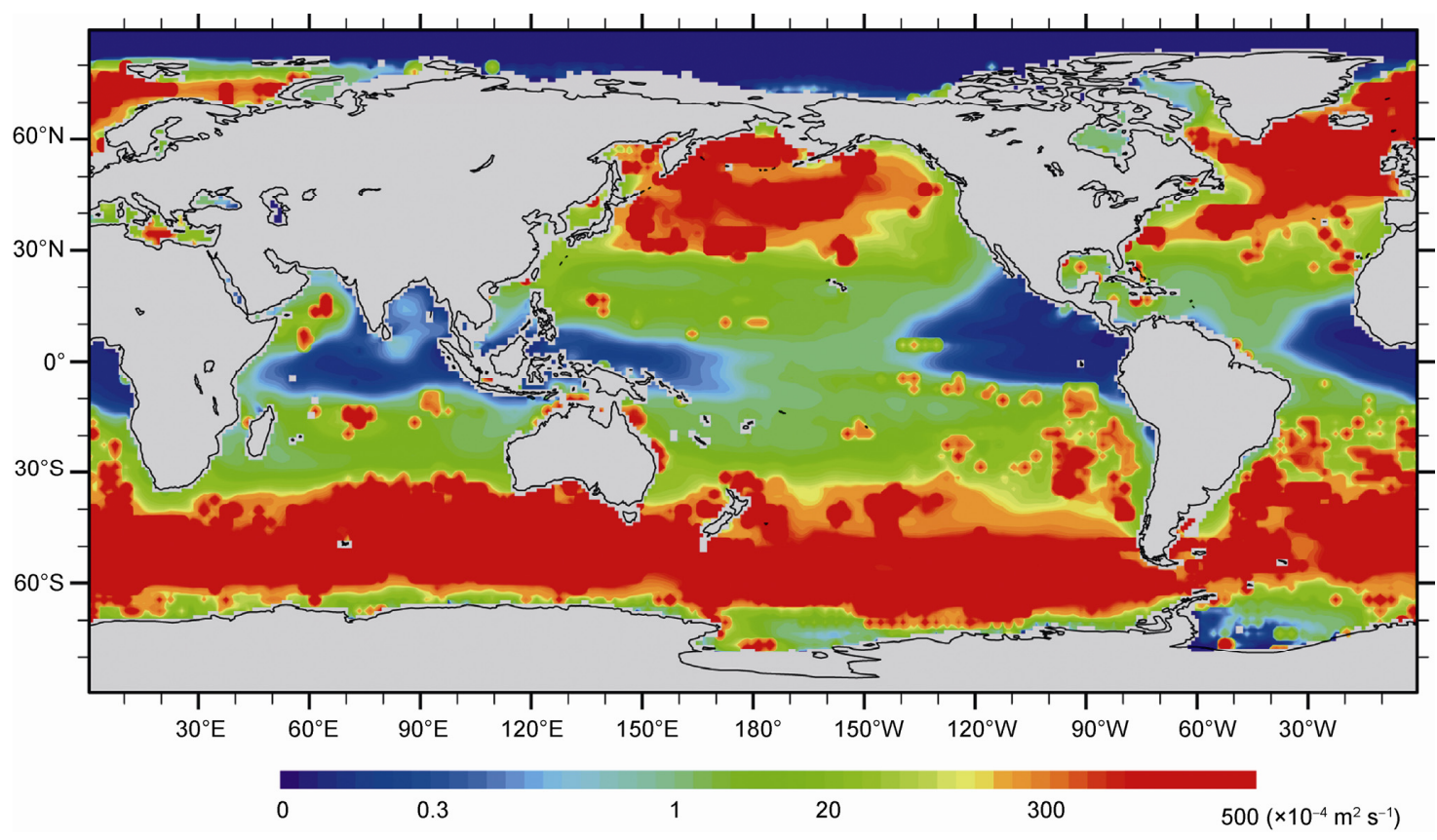

图 8 海洋上层(30 m 水深)风致湍流混合系数

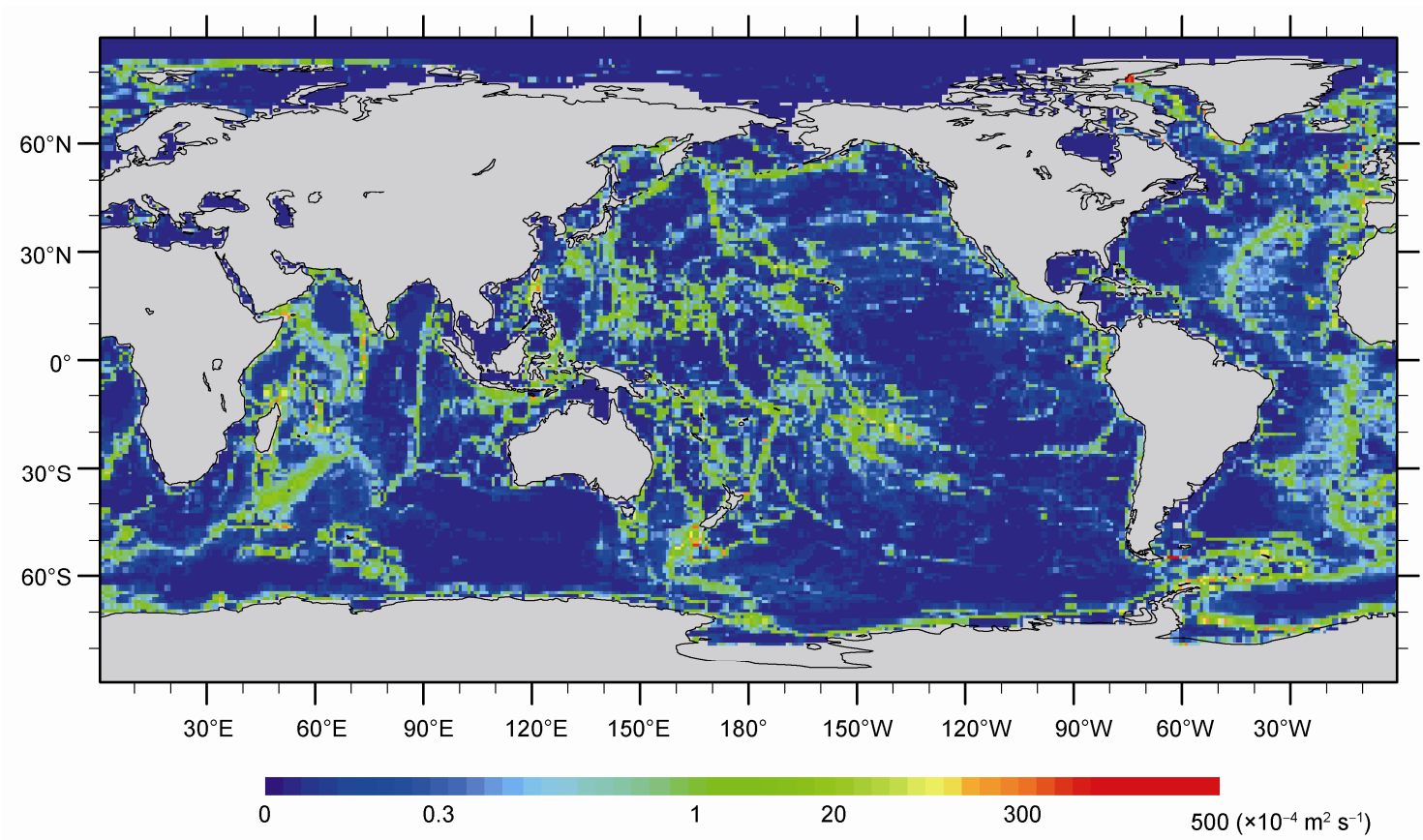

图 9 海底潮汐致湍流混合系数

此从图 9 可看出高混合系数主要分布在海底山脉附 近，南大西洋海山由于地质年龄较新，地形粗糙度大， 混合也比其他区域更剧烈, 一些较高大的海底山脉 甚至可将潮混合引入海洋上层(图 11).

混合系数的强度主要取决于湍流动能耗散的强
度和局地层结稳定性, 也即浮力频率的大小. 赤道地 区由于层结强而风输入能量小, 因此混合系数也小, 南大洋则相反. 由图 12 可知, 风能输入由低纬向南 北半球的中高纬逐渐增强, 北太平洋、北大西洋、南 太平洋都有很强的湍流动能耗散, 这和大气层中风 


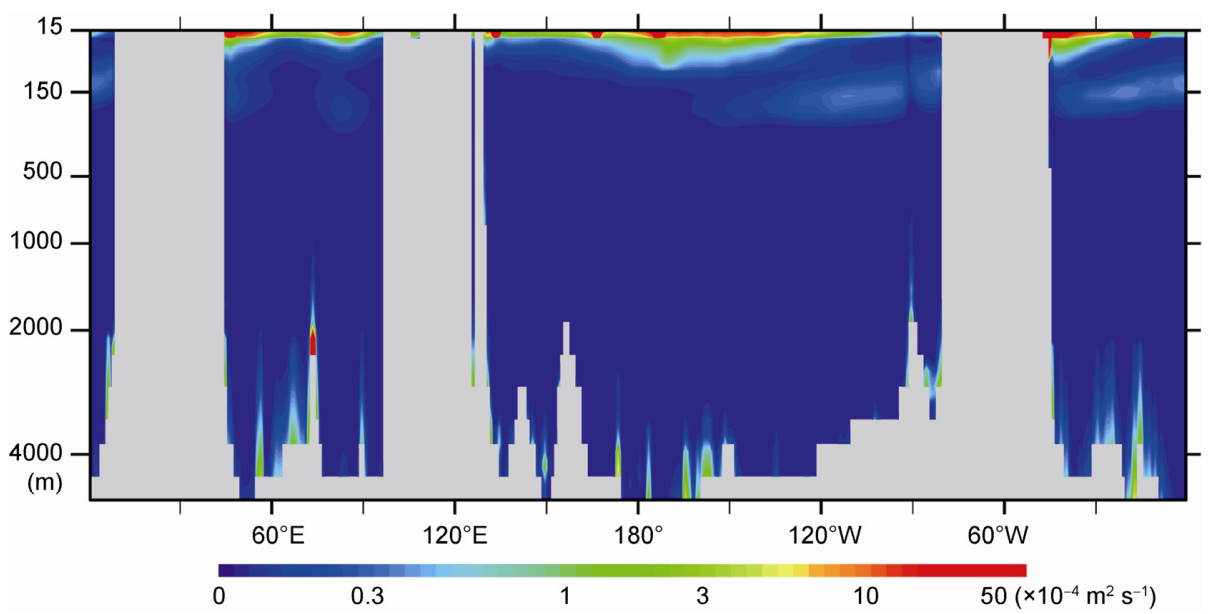

图 10 赤道地区大洋湍流混合系数垂直分布

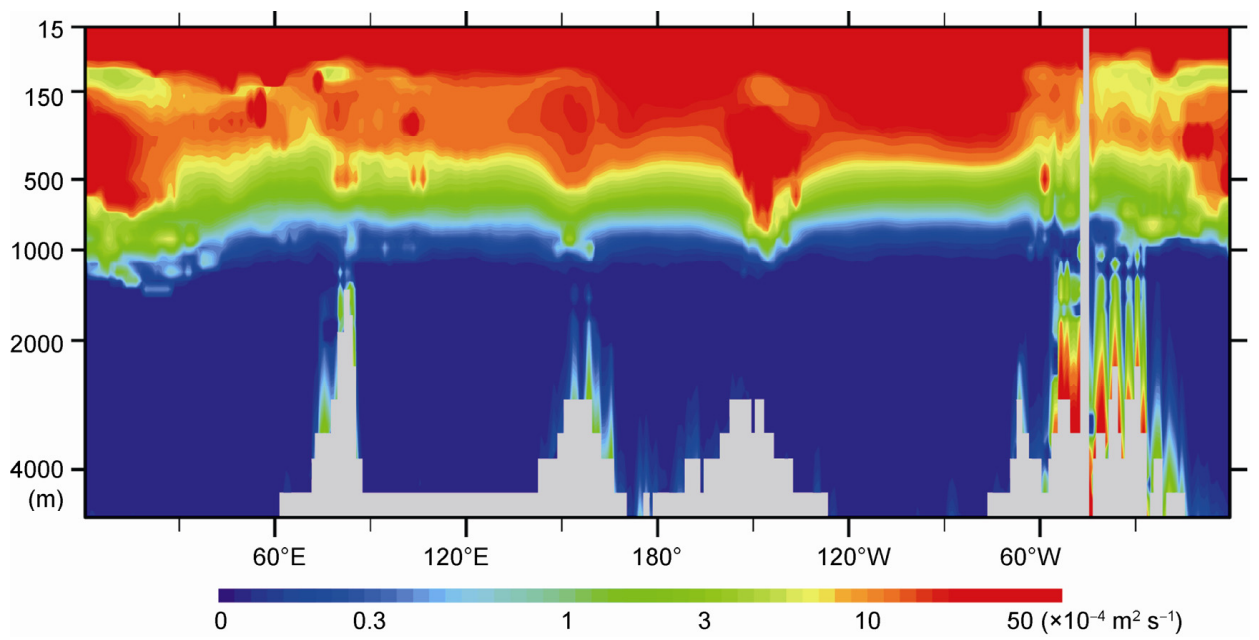

图 11 南大洋 $\left(60^{\circ} S\right)$ 湍流混合系数垂直分布

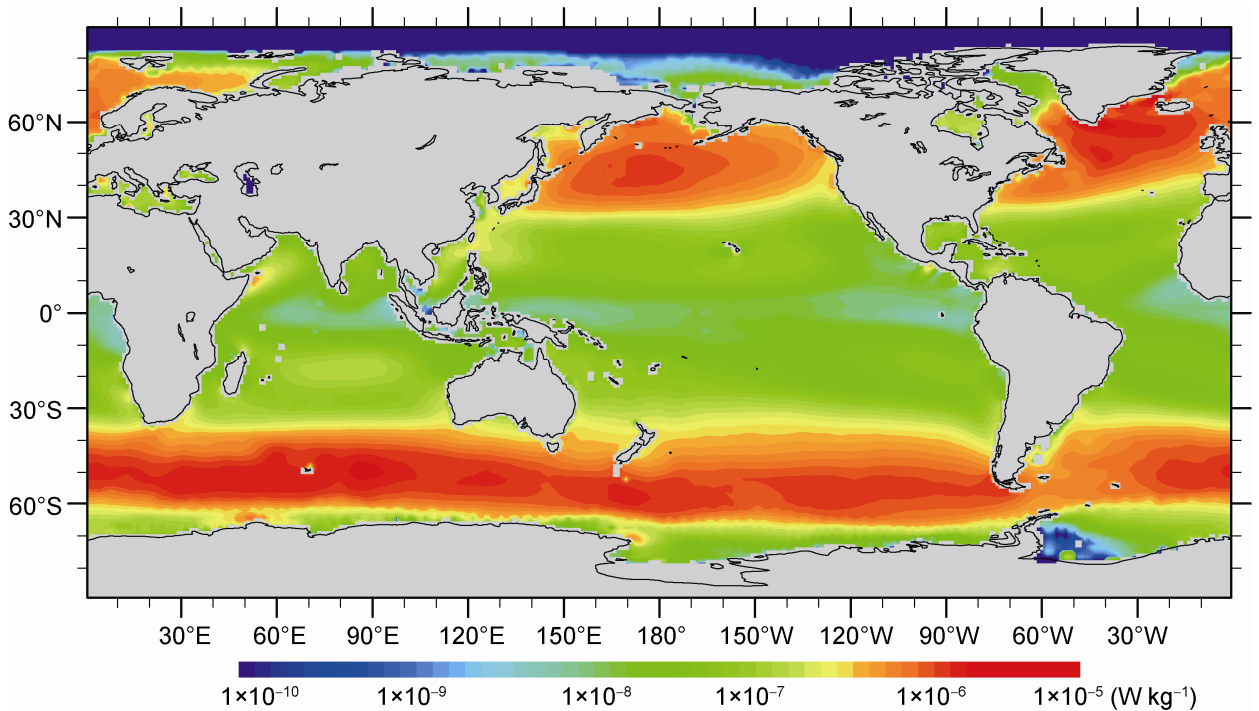

图 12 海洋上层 $(30 \mathrm{~m}$ 水深)风致湍流动能耗散 
的分布是一致的, 不过在北极地区由于海冰覆盖等 原因风能几乎无法输入到海洋中. 海洋中层结的分 布则几乎和风能相反, 赤道地区尤其是赤道太平洋 东岸和大西洋东岸层结非常强, 北太平洋和北大西 洋也有一定程度的稳定层结, 南大洋南极绕极流处 层结最弱(图 13). 因此层结和风能输入的空间分布共 同决定了中高纬和低纬巨大的混合系数差异, 也意 味着南大洋成为了全球风能输入的核心区域.

垂直方向上海洋中存在温跃层, 因此也存在层 结最强的区域, 这个区域也成为表层风输入能量向 下传播的障碍层. 在赤道地区障碍层非常显著, 并且 在大洋东部垂直上存在两个. 一个位于约 $100 \mathrm{~m}$ 水深
处, 另一个存在于约水深 $300 \mathrm{~m}$ 处(图 14). 这种分布 决定了混合系数也存在两个极值区，一个在 $100 \mathrm{~m}$ 水 深以上, 另一个约在 $150 \mathrm{~m}$ 处位于两个障碍层之间 (图 10). 南大洋的障碍层较弱且分布也比赤道宽, 主 要存在于 $150 \mathrm{~m}$ 水深以上(图 15). 由于南大洋的强风 能输入，这条弱障碍层带几乎不能有效的抑制能量 的向下传输, 因此该地区的风混合可以直达 $1000 \mathrm{~m}$ 水深处. 类似于赤道, 南大洋的障碍层也把混合极值 区分为两个部分，一个位于 $150 \mathrm{~m}$ 水深以上，另一个 位于水深 150 500 m(图 11). 但在障碍层极弱的南太 平洋地区二者已无法显著区分. 大洋深海的层结变 化很小，因此潮汐引起的混合效应基本上自海底向

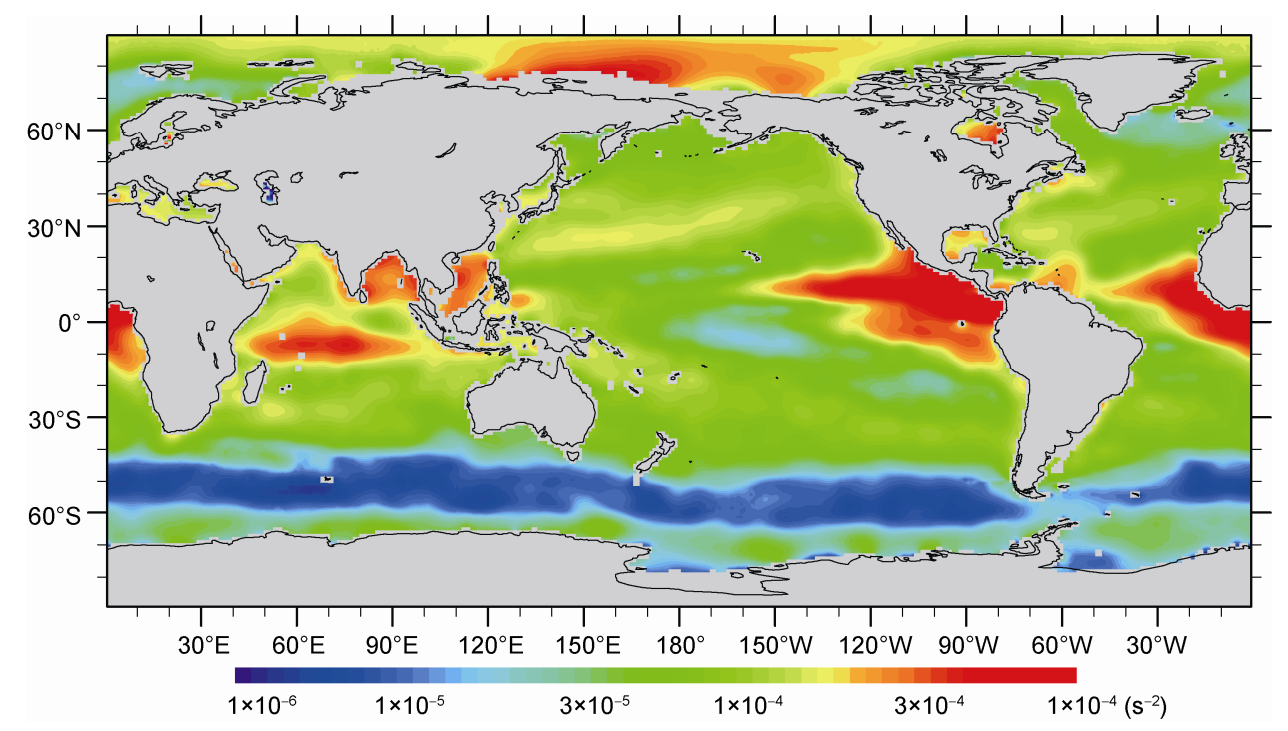

图 13 海洋上层 $(30 \mathrm{~m}$ 水深)浮力频率平方

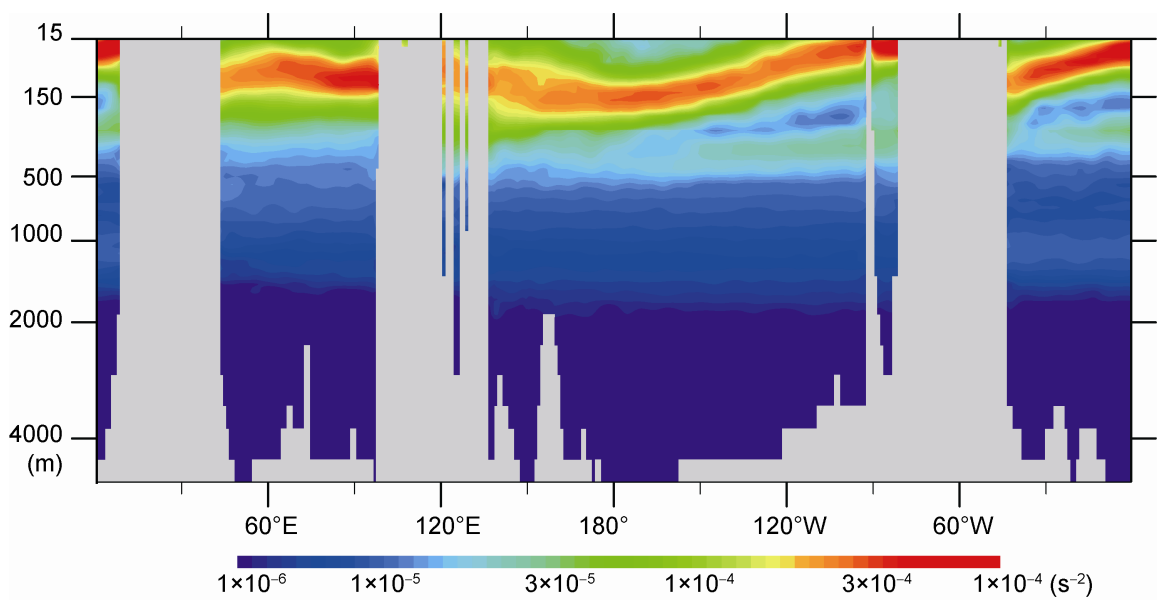

图 14 赤道浮力频率平方垂直分布 


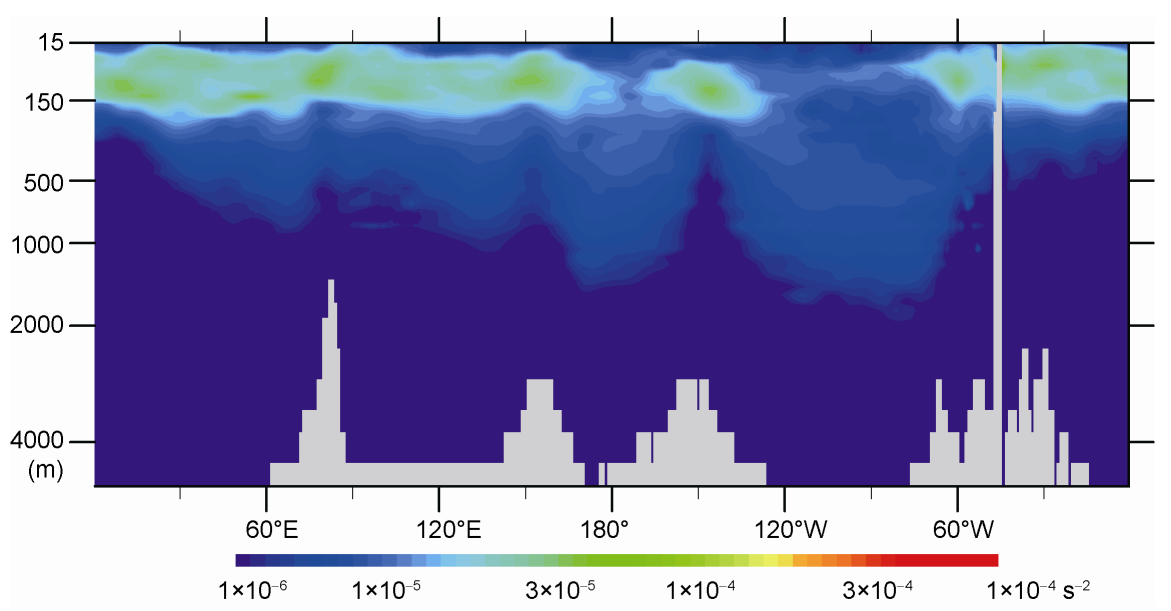

图 15 南大洋 $\left(60^{\circ} S\right)$ 浮力频率平方垂直分布

上呈指数形式衰减.

\section{6 结论}

本文系统讨论了一个包含风与潮汐作用的湍流 混合系数气候态数据集的建立过程及混合系数全球 分布特征, 讨论了一些计算方案对能量守恒的影响. 对全球风输入能量引起混合的参数化是本文的主要 内容, 潮汐引起混合的参数化主要引用 Simmons 等 (2004)和 Laurent 等(2002)的工作. 研究结果表明.

(1) 从能量守恒的角度考虑, 在海洋模式中引入 风输入的能量应尽可能的选用高时间分辨率的资料. 相对于 $6 \mathrm{~h}$ 分辨率资料, 用月平均化的资料会引起最 大约 $46.2 \%$ 的能量损失. 如果先用瞬时资料获得参数 化的混合系数, 再对瞬时混合系数做气候平均则能 够较好的保持总能量守恒, 但是能量的瞬时输入过 程则会被抹去, 因此气候态的混合系数数据集只适 用于年际尺度以上的长期气候数值积分使用.

(2) 参数化方案中常见的人为设定混合系数上 限的方法会引起极大的能量损失, 最高可达 $90 \%$, 远 超过使用平均化资料带来的损失. 此外, 人为限定还 会改变能量输入的自然周期律, 本文中热带地区风 输入的半年周期被混淆为半年和一年两种周期律. 因此在数值模式中用包含人为限定算法的参数化方 案后, 极有可能模拟出实际并不存在的虚假周期规 律, 并削弱真实的周期波动, 得出错误的研究结论.

(3) 不考虑外部能量的 $\mathrm{K}$ 理论参数化方案在能 量守恒量级和运动周期上都显著异于外部风输入的
特征. 以本文所举的 PP 方案为例, 其对热带大洋重 力位能平衡的贡献要比风致混合小 $90 \%$ 以上. 此外, PP 方案也模拟出实际并不存在的年变化周期律. 在 恰当调节参数的支持下，这类方案也许可以获得较 好的当前气候态模拟结果，但应对气候变化模拟则 缺乏足够的理论依据.

(4) 风引起的混合系数远远大于潮汐, 且在海洋 上层大致以温跃层为界呈现出分层结构. 南大洋深 厚的强混合区域使其成为了全球最重要的风能输入 海洋源地. 潮汐能输入主要集中于海洋地形粗䊁度 较高的地区，且随距海底的距离呈指数衰减.

在分析中我们还发现了一个现象，风输入的能 量倍乘能量转化率后, 恰好等于湍流增加的重力位 能. 事实上, 风参数化混合系数和混合引起重力位能 改变是两个完全不同的计算过程, 出现这样的结果 是一个巧合, 还是意味着在稳定层结下所有转化为 混合的能量都可以进一步转化为重力位能, 是值得 深入研究的问题.

直接在数值模式中应用本数据集可能会由于过 大的混合系数引起模式积分不稳定，此外本数据集 某种意义上是能量输入可能引起的最大混合系数, 因此在使用前用恰当的技术处理是必要的. 本文推 荐两种处理方案, 一种是人为限定混合系数上限值, 但可能会引起巨大的能量损失，一定程度上失去了 本数据集的能量守恒意义. 另一种是改进混合方案, 比如采用预算步的形式，如果过大的混合系数在一 个积分步长内引起了过度混合的现象(将稳定层结混 
合为不稳定层结), 则可跳过湍流混合直接平均化温 种影响，如何更好的设计参数化方案使之更符合能 盐量. 引入本数据集会对大洋环流数值模拟产生何 量守恒也是下一步要进行的研究.

致谢感谢 Woods Hole 海洋研究所的 Dr. S. Jayne 为我们提供了潮汐作用的湍流混合系数气候态数据, 感谢审稿专 家提出的宝贵意见.

\section{参考文献}

黄嘉佑. 2004. 气象统计分析与预报方法. 北京: 气象出版社. 220-225

黄瑞新. 1998. 论大洋环流的能量平衡. 大气科学, 22: 180-192

雷需, 杨海军. 2008. 海洋垂直混合系数对大洋环流影响的敏感性研究. 北京大学学报 (自然科学版), 6: 864-870

刘玲玲, 黄传江. 2008. 局地混合率变化对热带太平洋 SST 的影响. 中国海洋大学学报(自然科学版), 6: 883-886

张宇, 林一隼, 王辉. 2014. 垂直湍流输送对大洋的重力位能和混合过程的影响. 大气科学, 38: 838-844

Baumert H Z. 2005. A novel two-equation turbulence closure for high Reynolds numbers. Part B: spatially non-uniform conditions. In: Baumert S

A S, eds. Marine Turbulence, Theories, Observations and Models. Cambridge: Cambridge University Press. 31-43

Boos W R, Scott J R, Emanuel K A. 2004. Transient diapycnal mixing and the meridional overturning circulation. J Phys Oceanogr, 34: 334-341

Bryan F. 1987. Parameter sensitivity of primitive equation ocean general circulation models. J Phys Oceanogr, 17: 970-985

Charnock H. 1955. Wind stress on a water surface. Q J R Meteorolog Soc, 81: 639-640

Craig P D, Banner M L. 1994. Modeling wave-enhanced turbulence in the ocean surface layer. J Phys Oceanogr, 24: 2546-2559

Huang C J, Wang W, Huang R X. 2007. Climate variability in the equatorial pacific ocean induced by decadal variability of mixing coefficient. J Phys Oceanogr, 37: 1163-1176

Huang R X. 1999. Mixing and energetics of the oceanic thermohaline circulation. J Phys Oceanogr, 29: 727-746

Ivey G N, Imberger J. 1991. On the nature of turbulence in a stratified fluid. part i: The energetics of mixing. J Phys Oceanogr, 21: 650-658

Kocsis O, Prandke H, Stips A, et al. 1999. Comparison of dissipation of turbulent kinetic energy determined from shear and temperature microstructure. J Mar Syst, 21: 67-84

Large W G, McWilliams J C, Doney S C. 1994. Oceanic vertical mixing: A review and a model with a nonlocal boundary layer parameterization. Rev Geophys, 32: 363-403

Laurent L C S, Simmons H L, Jayne S R. 2002. Estimating tidally driven mixing in the deep ocean. Geophys Res Lett, 29: 21-1-4

Lozovatsky I D, Roget E, Fernando H J S, et al. 2006. Sheared turbulence in a weakly stratified upper ocean. Deep Sea Res Part I, 53: 387-407

Lozovatsky I D, Fernando H J. 2013. Mixing efficiency in natural flows. Philos Trans A Math Phys Eng Sci, $371: 20120213$

Mellor G L, Yamada T. 1982. Development of a turbulence closure model for geophysical fluid problems. Rev Geophys, 20: 851-875

Moum J N. 1996. Efficiency of mixing in the main thermocline. J Geophys Res, 101: 12057-12069

Oakey N S, Greenan B J W. 2004. Mixing in a coastal environment: 2. A view from microstructure measurements. J Geohys Res C, 109: 014

Osborn T R. 1980. Estimates of the local rate of vertical diffusion from dissipation measurements. J Phys Oceanogr, 10: 83-89

Pacanowski R C, Philander S G H. 1981. Parameterization of vertical mixing in numerical models of tropical oceans. J Phys Oceanogr, 11: $1443-1451$

Simmons H L, Jayne S R, Laurent L C S, et al. 2004. Tidally driven mixing in a numerical model of the ocean general circulation. Ocean Modelling, 6: 245-263

Samelson R M. 1998. Large-scale circulation with locally enhanced vertical mixing. J Phys Oceanogr, 28: 712-726

Soloviev A, Lukas R. 2003. Observation of wave-enhanced turbulence in the near-surface layer of the ocean during TOGA COARE. Deep Sea Res Part I, 50: 371-395

Terray E A, Donelan M A, Agrawal Y C, et al. 1996. Estimates of kinetic energy dissipation under breaking waves. J Phys Oceanogr, 26: $792-807$ 